\title{
Imaging cardiac sarcoidosis with FDG-PET: Take a look at the right side!
}

\author{
Fabien Hyafil, $\mathrm{MD}$, PhD, ${ }^{\mathrm{a}}$ François Rouzet, $\mathrm{MD}, \mathrm{PhD},{ }^{\mathrm{a}}$ and Dominique Le \\ Guludec, MD, PhD \\ a Department of Nuclear Medicine, Centre Hospitalier Universitaire Bichat, Assistance Publique - \\ Hôpitaux de Paris, Inserm 1148, Université Paris Diderot, Paris, France
}

Received Jan 26, 2019; accepted Jan 28, 2019

doi: $10.1007 / \mathrm{s} 12350-019-01645-8$

See related article, pp. 2135-2143

\section{DETECTING CARDIAC SARCOIDOSIS}

Sarcoidosis is a multisystemic inflammatory granulomatous disease of unknown etiology. The clinical course of cardiac sarcoidosis varies from a benign to life-threatening disease leading to severe heart failure or sudden cardiac death. Cardiac symptoms are described in about $10 \%$ of patients with systemic sarcoidosis, ${ }^{1}$ but autopsy studies found a prevalence of $20 \%$ of cardiac lesions in the United States and Europe ${ }^{2}$ up to $50 \%$ in Japan, ${ }^{3}$ suggesting that cardiac sarcoidosis (CS) might be underdiagnosed. Active sarcoid granulomas secrete locally high levels of cytokines causing myocardial injuries and reducing perfusion through vasoconstriction and compression of the microvasculature. ${ }^{4}$ If left untreated, active sarcoid granulomas provoke the formation of interstitial fibrotic scars and lead ultimately to the development of irreversible dilated cardiomyopathy. The presence of inflammatory and fibrotic lesions in the heart can also generate severe ventricular arrhythmia or high-grade atrioventricular block with a risk of sudden death. It is therefore important to identify cardiac localization of sarcoidosis at an early stage to prevent the onset of severe acute and chronic complications of $\mathrm{CS}$ in this often young population of patients. An important challenge for the detection of CS is that the

Reprint requests: Fabien Hyafil, MD, PhD, Department of Nuclear Medicine, Bichat University Hospital, 46 rue Henri Huchard, 75018 Paris, France; fabien.hyafi@aphp.fr

J Nucl Cardiol 2020;27:2144-8.

$1071-3581 / \$ 34.00$

Copyright (c) 2019 American Society of Nuclear Cardiology. disease is distributed heterogeneously in the heart. Endomyocardial biopsies (EMB) showing the presence of non-caseating granulomas on histology is the gold standard for the diagnosis of CS but has only a sensitivity of $20 \%$. Several scores formed of a combination of criteria integrating the presence of abnormalities observed on the ECG, echocardiography, cardiac magnetic resonance imaging (MRI), nuclear imaging, and the results of histology on EMB have been proposed to improve the sensitivity of CS detection. The most commonly used are the score of the Japanese Ministry of Health and Welfare (JMHW) revised in $2006^{5}$ and of the Heart Rhythm Society (HRS), ${ }^{6}$ but the diagnostic performance of each score cannot be reliably estimated in absence of gold standard. These scores have not also demonstrated prospectively their value for the prediction of clinical events.

\section{INCREASING ROLE OF NON-INVASIVE IMAGING IN CARDIAC SARCOIDOSIS}

In patients with a suspicion of CS and arrhythmia on ECG, echocardiography is the first-line imaging tool to search for abnormalities in myocardial wall thickness or contraction. ${ }^{7}$ However, echocardiography lacks sensitivity for the detection of subtle changes observed in CS and its findings are frequently non-specific. Cardiac MRI provides a higher sensitivity than echocardiography to assess the locations and extent of cardiac sarcoidosis. In addition to the analysis of segmental myocardial contraction with cine sequences, cardiac MRI allows for the identification of oedema on T2weighted sequences, and oedema and fibrosis on late gadolinium enhancement (LGE) sequences. Typical aspects in favor of CS on MRI are the presence of nodular enhancement on LGE sequence with a patchy distribution and segmental wall motion abnormalities on cine sequences that do not have a typical distribution of 


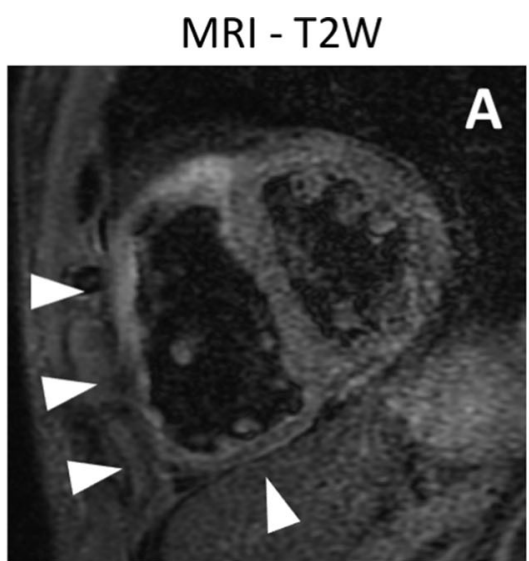

Baseline

+3 months

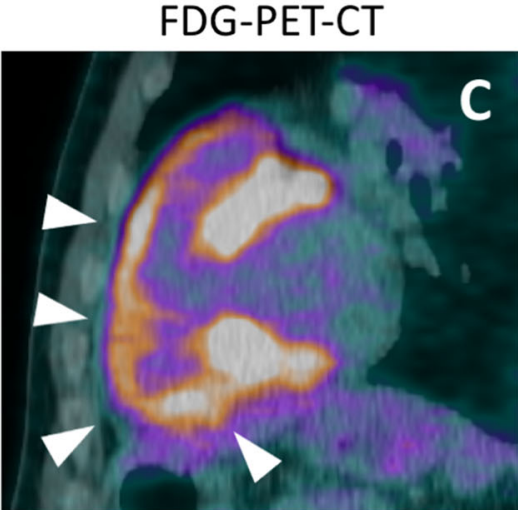

FDG-PET-CT

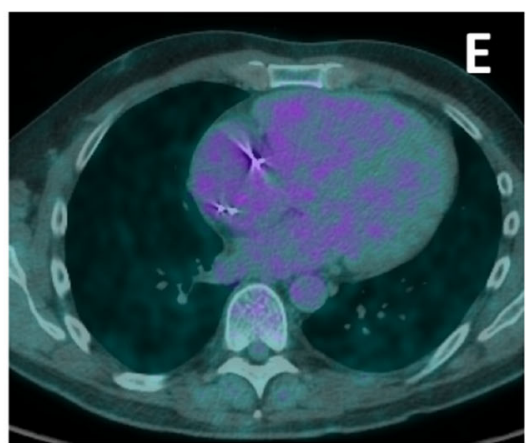

FDG-PET-CT

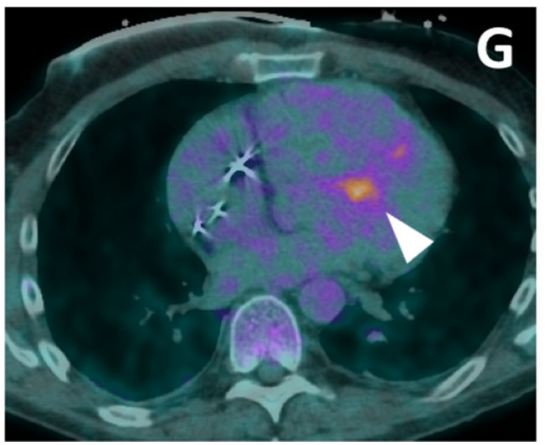

MRI - DCE

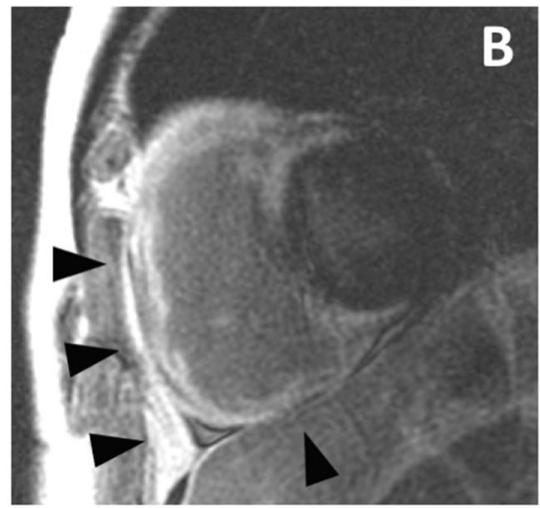

FDG-PET (MIP)

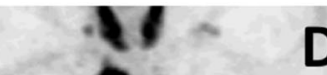

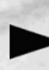
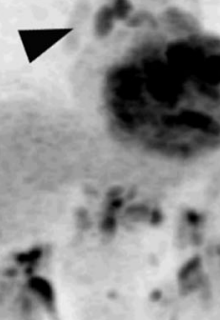

FDG-PET (MIP)

$\mathbf{F}$

FDG-PET (MIP)

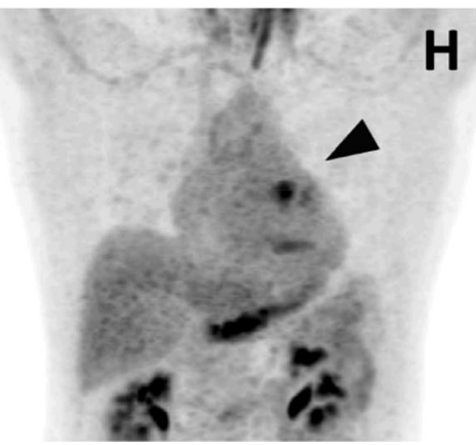


4Figure 1. Cardiac sarcoidosis involving the right ventricle (RV) assessed with multimodality imaging. This patient was admitted for a first episode of ventricular tachycardia. Echocardiography showed moderate dilatation and dysfunction of the RV. Cardiac MRI evidenced the presence of a discrete hyperintensity in the free wall of the RV on T2-weighted (T2W) sequences (A, arrowheads) associated with delayed contrast enhancement (DCE) on the inversion recovery sequence (B, arrowheads). FDG-PET imaging was acquired after patient preparation with a high-fat low-carb diet to search for an inflammatory process in the heart and showed intense FDG uptake in the right ventricle extending into the interventricular septum (C, arrowheads), whereas no FDG signal was detected in the remaining left ventricle confirming the successful suppression of FDG uptake in the myocardium. On maximum intensity projection (MIP) reconstruction of whole-body PET acquisition, high FDG uptake was also detected in peri-bronchial lymph nodes (D, white arrowheads), which were biopsied and contained sarcoid granuloma on histology. Endomyocardial biopsy was not performed in these patients as the probability of active cardiac sarcoidosis was deemed high. Steroid therapy was started and an ICD implanted. On the second FDG-PET acquisition performed 3 months after the initiation of immunosuppressive agents $(\mathbf{E}$, F), note the complete regression of the FDG signal in the heart and in mediastinal lymph nodes. One year later, the patient received a shock from the ICD. Internal ECG memory of the ICD showed an episode of sustained VT. FDG-PET imaging was repeated and evidenced the appearance of a new FDG signal in the interventricular septum in favor of CS relapse $(\mathbf{G}$, $\mathbf{H}$, arrowheads).

coronary perfusion territories. ${ }^{8}$ Nonetheless, the sensitivity of T2-weighted sequences is relatively low for the detection of edema, and the imaging aspects observed on LGE sequences lack specificity for cardiac sarcoidosis. Nuclear imaging offers to identify the presence of inflammatory activity in granulomas with high sensitivity. $^{9} \quad$ Nuclear techniques can thus provide complementary information to morphological imaging with the possibility to identify inflammatory processes associated with sarcoidosis activity. Activated macrophages and $\mathrm{CD} 4+\mathrm{T}$ lymphocytes present in active granulomatous sarcoid lesions have high glycolytic activity to sustain their energy demands and accumulate FDG. FDG-PET has demonstrated its high sensitivity for the detection of active granulomas in sarcoidosis and has now replaced in most centers SPECT with ${ }^{67}$ Galliumcitrate. ${ }^{11}$ Whole-body acquisitions with FDG-PET offer also to identify extracardiac extension of sarcoidosis that are more accessible than the myocardium for biopsy. The detection of active granulomas in the heart requires, however, to prepare patients with a dedicated diet before imaging to suppress FDG uptake in the normal myocardium. The optimal protocol consists of a lowcarbohydrate high-fat meal the day before imaging followed by an extended fasting ( $>18$ hours). When FDG uptake in the normal myocardium is completely suppressed, the presence of multi-focal FDG signals in the heart corresponds to regions of active inflammation and, in patients with a clinical suspicion of sarcoidosis, is highly suggestive of active granulomas. Complete suppression of the physiological FDG uptake is hard to achieve in all patients. In particular, moderate myocardial FDG uptake can persist in the basal segments of the lateral wall and in regions that have been submitted to transient ischemia. The addition of myocardial perfusion imaging to FDG acquisitions is now recommended and helps improve the specificity of PET interpretations. ${ }^{10}$ The combination of high FDG uptake and low rest perfusion in the heart on PET is highly suggestive of the presence of active granulomas and has been associated with a worse prognosis in patients with $\mathrm{CS} .{ }^{11}$ The availability of these new imaging modalities has significantly improved the detection of CS.

\section{WHICH ROLE FOR ENDOMYOCARDIAL BIOPSY AT THE TIME OF FDG-PET IMAGING?}

The study of Omote et al. ${ }^{12}$ published in this issue of the Journal of Nuclear Cardiology questions whether EMB has still a role for the diagnostic of CS at the time of FDG-PET imaging. In this retrospective study, the authors found that, in the absence of FDG uptake in the RV on PET, the positive rate of endomyocardial biopsy (EMB) in the diagnosis of cardiac sarcoidosis was very low $(6 \%)$. In addition, localization of sarcoidosis in organs or lymph nodes that are more accessible than the heart for biopsy could be evidenced in most patients on FDG-PET imaging. The results of this study question whether it is still worth to submit patients with a suspicion of CS and no FDG uptake in the RV to the risks of EMB-related complications for such a low diagnostic yield. A surprising finding in the study of Omote et al. $^{12}$ is that the association between the positivity of EMB and the presence of FDG uptake was stronger for the RV than for septal segments, in which EMB is performed. In fact, high FDG uptake was also present in septal segments, but the septal FDG signal might be less specific for the presence of granulomatous lesions than the RV signal. Another explanation for these results suggested by Omote et al. ${ }^{12}$ is that the presence of FDG uptake in the RV might be a marker of the extension and severity of cardiac sarcoidosis. Hence, the association of histology-confirmed extracardiac sarcoidosis and high FDG uptake in the RV on PET identifies a group of patients with high probability of CS who is likely to benefit from the initiation of immunosuppressive therapy. At the time of FDG-PET imaging, indications for EMB in patients of a suspicion of CS will 
thus likely decrease with, on one hand, the identification of a group of patients with high FDG signal in the RV who have a high probability of CS and an indication to immunosuppressive treatment and, on the other hand, a group of patients with no FDG signal in the RV, in whom the diagnostic yield of EMB is low.

\section{RISK STRATIFICATION WITH MULTIMODALITY IMAGING: HOW FAR CAN WE GO?}

Adequate risk stratification is even more important than the diagnosis of CS. The main goal of CS imaging is to identify with high accuracy the group of patients with the highest risk of clinical events who will benefit the most from immunosuppressive agents and of pacemaker (PM) and/ or implantable cardiac defibrillators (ICD). During the past 10 years, several new prognostic imaging markers have been identified for CS. On cardiac MRI, the presence of focal regions of delayed enhancement on LGE sequences has been associated with a worse prognosis in patients with $\mathrm{CS} .{ }^{13}$ On PET, high FDG uptake in the RV has been associated with an increased risk of death or sustained ventricular tachycardia at follow-up in patients with CS. ${ }^{11}$ The prognostic value of this marker has been confirmed in the study of Omote et al. ${ }^{12}$ In the $\mathrm{LV}$, the association of perfusion defects on rest myocardial perfusion imaging with high FDG uptake was associated with an increased risk of clinical events in comparison to the high FDG signal alone. ${ }^{11}$ Hence, non-invasive imaging allows us to identify two groups of patients: a group with one of these imaging markers at high risk of cardiac events and who will likely benefit from immunosuppressive agents and a group with none of these imaging markers at low risk of cardiac events. Nevertheless, an important proportion of patients imaged for a suspicion of CS presents only moderate FDG alone in the LV with no perfusion defect that may be related either to early active sarcoid lesions or to benign inflammatory reactions and non-specific FDG uptake in the myocardium. The clinical management of this group of patients is challenging in the absence of gold standard. The availability of PET tracers that are more specific than FDG for the identification of active granulomatous sarcoid lesions ${ }^{14}$ and the combined analysis of PET and MRI acquisitions ${ }^{15}$ might allow for better distinction between early sarcoid lesions and non-specific imaging aspects observed in the heart.

\section{PHENOTYPING CARDIAC SARCOIDOSIS WITH MULTIMODALITY IMAGING}

Recent advances in the non-invasive imaging of CS illustrate nicely how a multimodality approach can help assessing a cardiac disease in several ways. First, the integration of the results of different imaging modalities that separately lack specificity results into a more accurate detection of the different components of CS. Second, the presence, extent, and severity of a disease can be assessed more precisely resulting into better risk stratification and selection of patients for treatments, as well as providing useful tools for assessing the efficacy of immunosuppressive treatments (Figure 1). Nevertheless, several questions remain regarding the role of imaging in the clinical management of patients with CS. Which patients should be screened for CS? Which patients should be treated by steroids and for how long? Which patients should be implanted with PM or ICD? What is the role of imaging in assessing the efficacy of immunosuppressive agents in patients with CS? The accumulation of data from large cohorts of patients with CS will help answer these questions and define the role of multimodality imaging in the risk stratification and monitoring of patients with CS. The multimodality imaging approach that has been developed for the evaluation of CS might also apply for the phenotyping of other cardiac diseases such as amyloidosis, aortic valve stenosis, or atherosclerosis and play an important role in the development of personalized medicine in the cardiovascular field.

\section{Disclosure}

F Hyafil, F. Rouzet, and D. Le Guludec have nothing to disclose in relation to this Editorial.

\section{References}

1. Iannuzzi MC, Rybicki BA, Teirstein AS. Sarcoidosis. N Engl J Med 2007;357:2153-65.

2. Silverman KJ, Hutchins GM, Bulkley BH. Cardiac sarcoid: a clinicopathologic study of 84 unselected patients with systemic sarcoidosis. Circulation 1978;58(6):1204-11.

3. Iwai K, Sekiguti M, Hosoda Y, et al. Racial difference in cardiac sarcoidosis incidence observed at autopsy. Sarcoidosis 1994;11(1):26-31.

4. Le Guludec D, Menad F, Faraggi M, Weinmann P, Battesti JP, Valeyre D. Myocardial sarcoidosis. Clinical value of technetium99m sestamibi tomoscintigraphy. Chest 1994;106(6):1675-82.

5. Hiraga H, Yuwai K, Hiroe M. Diagnostic standard and guidelines for sarcoidosis [in Japanese]. Jpn. J Sarcoidosis Other Granulomatous Disord 2007;27:89-102.

6. Birnie DH, Sauer WH, Bogun F, et al. HRS expert consensus statement on the diagnosis and management of arrhythmias 
associated with cardiac sarcoidosis. Heart Rhythm 2014;11(7):1305-23.

7. Slart R, Glaudemans A, Lancellotti $P$, et al. A joint procedural position statement on imaging in cardiac sarcoidosis: from the Cardiovascular and Inflammation \& Infection Committees of the European Association of Nuclear Medicine, the European Association of Cardiovascular Imaging, and the American Society of Nuclear Cardiology. J Nucl Cardiol 2018;25(1):298-319.

8. Greulich S, Deluigi CC, Gloekler S, et al. CMR imaging predicts death and other adverse events in suspected cardiac sarcoidosis. JACC Cardiovasc Imaging 2013;6(4):501-11.

9. Piekarski E, Benali K, Rouzet F. Nuclear imaging in sarcoidosis. Semin Nucl Med 2018;48(3):246-60.

10. Chareonthaitawee P, Beanlands RS, Chen W, et al. Joint SNMMIASNC expert consensus document on the role of (18)F-FDG PET/ $\mathrm{CT}$ in cardiac sarcoid detection and therapy monitoring. J Nucl Cardiol 2017;24(5):1741-58.

11. Blankstein R, Osborne M, Naya M, et al. Cardiac positron emission tomography enhances prognostic assessments of patients with suspected cardiac sarcoidosis. J Am Coll Cardiol 2014;63(4):32936.
12. Omote K, Naya M, Koyanagawa K, et al. (18)F-FDG uptake of the right ventricle is an important predictor of histopathologic diagnosis by endomyocardial biopsy in patients with cardiac sarcoidosis. J Nucl Cardiol 2019. https://doi.org/10.1007/s12350018-01541-7.

13. Murtagh G, Laffin LJ, Beshai JF, et al. Prognosis of myocardial damage in sarcoidosis patients with preserved left ventricular ejection fraction: risk stratification using cardiovascular magnetic resonance. Circ Cardiovasc Imaging 2016;9(1):e003738.

14. Pizarro C, Kluenker F, Dabir D, et al. Cardiovascular magnetic resonance imaging and clinical performance of somatostatin receptor positron emission tomography in cardiac sarcoidosis. ESC Heart Fail 2018;5(2):249-61.

15. Wicks EC, Menezes LJ, Barnes A, et al. Diagnostic accuracy and prognostic value of simultaneous hybrid $18 \mathrm{~F}$-fluorodeoxyglucose positron emission tomography/magnetic resonance imaging in cardiac sarcoidosis. Eur Heart $\mathrm{J}$ Cardiovasc Imaging 2018;19(7):757-67.

Publisher's Note Springer Nature remains neutral with regard to jurisdictional claims in published maps and institutional affiliations. 